\title{
Unsteady Aerodynamics of Low Reynolds Number Flight
}

\author{
Luis P Bernal ${ }^{1}$ and Huai-Te $\mathrm{Yu}^{2}$ \\ University of Michigan, Ann Arbor, Michigan, 48109-2140
}

\begin{abstract}
Small autonomous or remotely piloted air vehicles offer new capabilities in agility and maneuverability. A variety of missions have been proposed for these vehicles that require aggressive maneuvers and unique take-off and landing capabilities. From an unsteady aerodynamics point of view these capabilities expand the parameter space toward the high rotation rate and acceleration, and low Reynolds number region of the envelope. In this paper we review recent experimental results, numerical simulations and theoretical analysis of relevant unsteady aerodynamics problems. It is shown that unsteady effects can significantly increase lift generation during maneuvers. Vortex dynamics and other relevant physical processes which contribute to unsteady force generation will be discussed. Simple theoretical models that provide reasonably accurate estimates of unsteady aerodynamic forces are reviewed.
\end{abstract}

\section{Nomenclature}

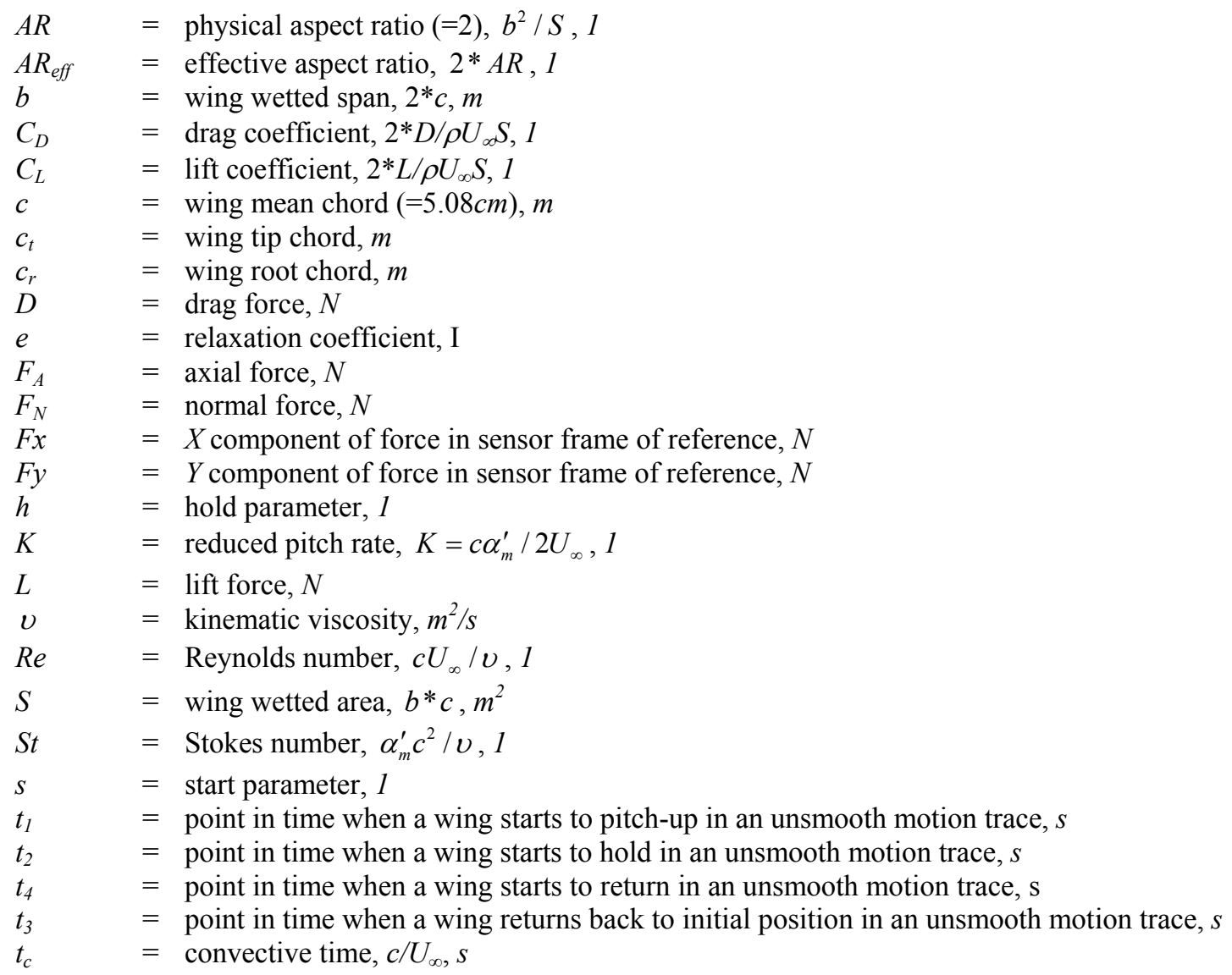

${ }^{1}$ Professor, Department of Aerospace Engineering, University of Michigan, 1320 Beal Avenue, Ann Arbor, MI 48109-2140, 1pb@umich.edu, AIAA Senior Member.

${ }^{2}$ Graduate Research Assistant, Department of Aerospace Engineering, University of Michigan, 1320 Beal Avenue, Ann Arbor, MI 48109-2140, htyu@umich.edu, AIAA Student Member. 


$\begin{array}{ll}t_{p} & =\text { pitch time, } \alpha_{m} / \alpha_{m}^{\prime}, s \\ U_{\infty} & =\text { free-stream velocity, } \mathrm{m} / \mathrm{s} \\ \alpha(t) & =\text { angle of attack, } \mathrm{deg} \\ \alpha_{m} & =\text { maximum angle of attack, } \mathrm{deg} \\ \alpha^{\prime}(t) & =\text { pitch rate in time, } \mathrm{deg} / \mathrm{s} \\ \alpha_{m}^{\prime} & =\text { maximum pitch rate, } \mathrm{deg} / \mathrm{s} \\ \alpha_{m}^{\prime \prime} & =\text { maximum pitch acceleration, } \mathrm{deg} / \mathrm{s}^{2}\end{array}$

\section{Introduction}

$\mathrm{T}$ HE development of small autonomous and remotely piloted air vehicles that can fly in strong gusts and air turbulence, have long endurance and perch requires a better understanding of the unsteady aerodynamics of low-Reynolds-number flight. Biological flyers offer examples of remarkable agility and maneuverability. In a typical hunting maneuver birds are able to slow down to the prey speed, capture it, and quickly accelerate to their preferred flight conditions. These maneuvers are performed with remarkable precision and maintaining accurate control of body position to be able to capture the prey. Small birds and insects also show remarkable agility in response to gusts and to maintain controlled flight for feeding. Birds and large insects operate at low Reynolds numbers in the range 1,000 to less than 100,000. Engineered small air vehicles today do not have comparable agility or maneuverability, which is desirable for a number of missions. Of considerable interest are rapid pitching maneuvers and the large aerodynamic forces that could be generated during the motion. Birds are able to rotate their wings in few convective times to slow down in very short distances, which results in large nondimensional reduced pitch rates compared to values found in manned aircraft performing post-stall maneuvers, for example. In this paper we review recent results on the unsteady aerodynamics of low-Reynolds-number high-pitch-rate motions of low aspect ratio wings. The pitch motion is large amplitude resulting in the formation leading edge, trailing edge and tip vortices. Wings of different planform geometry are considered and the effect of pivot axis is studied. Of considerable interest is to document the formation of leading edge, trailing edge and tip vortices during the wing motion, the associated time scales, as well as the relation between vortex and force development.

In the present paper we highlight some recent results on the unsteady aerodynamics of low-aspect-ratio flat-plate wings based on the results reported by $\mathrm{Yu}$ et $a l^{1}$, $\mathrm{Yu}$ and $\mathrm{Bernal}^{2}$ and $\mathrm{Yu}^{3}$ For additional comprehensive details the reader is referred to those paper, and more specifically to reference 3 , we a extensive review of the relevant literature is provided. We consider a constant rate pitching motion from 0 to 45 degrees angle of attack of flat plate wings with effective aspect ratio 4, several planform geometries and pivot axis. For a given wing geometry, maximum pitch angle, pivot location, there are two independent parameters that define the flow: free stream velocity and wing pitch rate. Normalizing both parameters with relevant combinations of wing chord and kinematic viscosity gives Reynolds number $\left(U_{\infty} c / v\right)$ and Stokes number $\left(\alpha_{m}^{\prime} c^{2} / v\right)$, shown as coordinates in Figure I. The abscissa is Reynolds number, which is a ratio of flow advection time to viscous diffusion time; the ordinate is Stokes number, which is a ratio of pitch-rate characteristic time to viscous diffusion time.

$$
K=\frac{c \alpha_{m}^{\prime}}{2 U_{\infty}}=\frac{S t}{2 \operatorname{Re}}=\frac{\alpha_{m} t_{c}}{2 t_{p}}=\frac{\alpha_{m}}{2 \tau}
$$

An important parameter for flow dynamics is the reduced pitch rate $K$ given by Eq. (1), which is the ratio of convective time to pitch-rate characteristic time. In Figure I. constant reduced pitch rate conditions form straight lines through the origin with increased slope as reduced pitch rate increases. Other characteristic times of interest are the pitch time $t_{p}=\alpha_{m} / \alpha_{m}$ 'and the convective time $t_{c}=c / U_{\infty}$; the ratio of $t_{p}$ to $t_{c}$ is denoted by $\tau$.

Figure I. shows experimental conditions considered in this study. Experiments were conducted at four freestream velocities $\left(U_{\infty}\right): 0 \mathrm{~cm} / \mathrm{s}(R e=0 \mathrm{k}), 8.6 \mathrm{~cm} / \mathrm{s}(R e \sim 4.3 \mathrm{k}), 17.5 \mathrm{~cm} / \mathrm{s}(R e \sim 8.9 \mathrm{k})$, and $25.6 \mathrm{~cm} / \mathrm{s}(R e \sim 13 \mathrm{k})$, and five pitch rates: $12.5^{\circ} / \mathrm{s}(S t \sim 0.5 \mathrm{k}), 25.6^{\circ} / \mathrm{s}(S t \sim 1.1 \mathrm{k}), 37.5^{\circ} / \mathrm{s}(S t \sim 1.7 \mathrm{k}), 76.4^{\circ} / \mathrm{s}(S t \sim 3.4 \mathrm{k})$, and $155^{\circ} / \mathrm{s}(S t \sim 7.0 \mathrm{k})$. In Figure I., square symbols represent the use of only rectangular wing; triangle symbols represent the use of several wing planforms. The wings were pitched at three pivot locations from $0^{\circ}$ to $45^{\circ}$, i.e. leading edge, mid-chord, and trailing edge. The ratio of pitch time to convective time is also presented in parenthesis in Figure I.; they vary from $\tau$ $=1$ to 18 . These correspond to unsteady flow $K>0$. Steady flow measurements, $K=0$, at a Reynolds number of $9 \times 10^{3}$ were also considered to quantify the steady flow around the wings; the corresponding point is shown on the horizontal axis. 


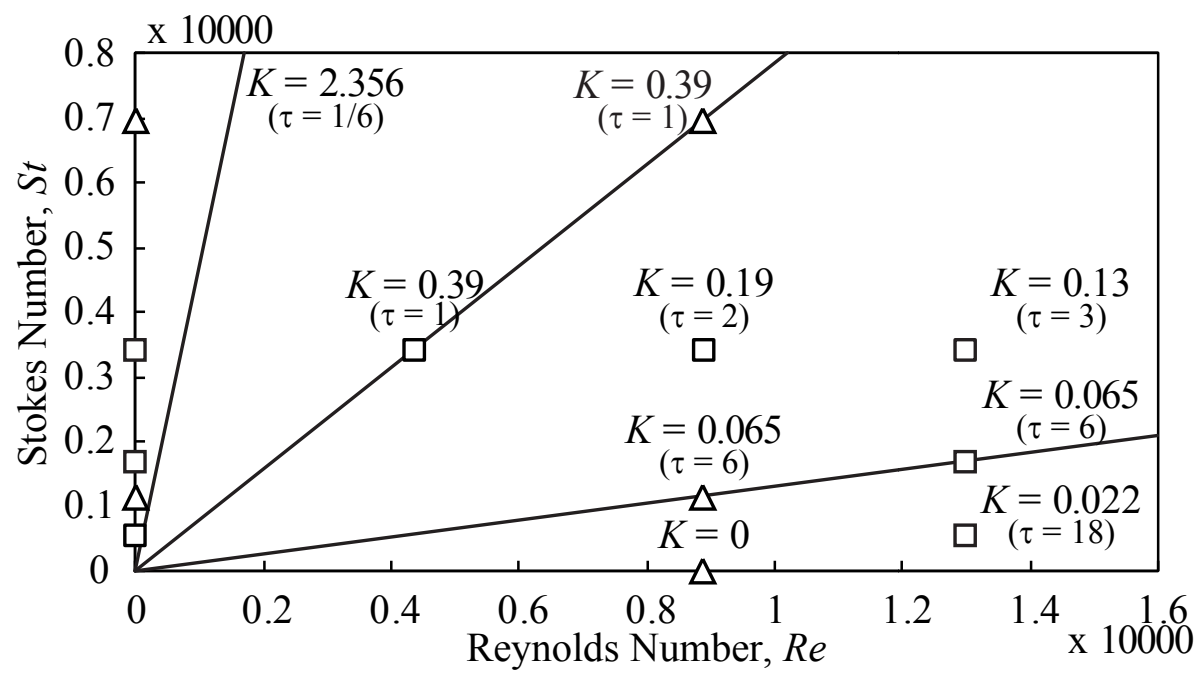

Figure I. Test cases of interest in St-Re parameter space.

\section{Flow Apparatus and Experimental Techniques}

\section{A. Wing Configuration and Water Tunnel}

The experiments were conducted in the Low-Turbulence Free Surface Water Channel at the University of Michigan, Department of Aerospace Engineering. The tests section is $0.6 \mathrm{~cm} \times 0.6 \mathrm{~cm} \times 2 \mathrm{~m}$. The steady free-stream velocity was controlled through an $\mathrm{AC}$ induction motor and the water depth which is carefully monitored during the experiment, and ranges from $5 \mathrm{~cm} / \mathrm{s}$ to $40 \mathrm{~cm} / \mathrm{s}$. A picture of the facility is shown in Figure 2 . The wings used in the present experiments were made of plexiglass sheet, and have two-inch chord and two chords immersed in water. The wing thickness is 0.125 ", which equals to $6.25 \%$ of chord; all edges are rounded. The wing installation is illustrated in Figure 3.

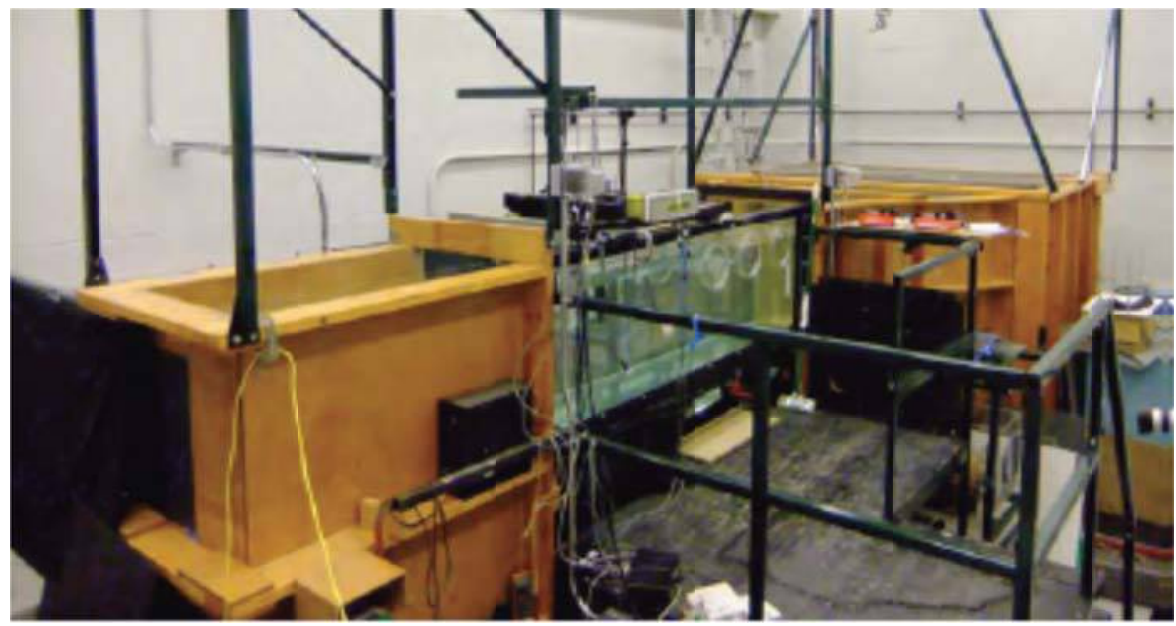

Figure 2. University of Michigan Low Turbulence Water Channel at the Department of Aerospace Engineering

\section{B. Wing Kinematics}

The wing motion is a linear pitch ramp from $0^{\circ}$ to $45^{\circ}$ with smoothing motion at the start and the end of the pitch ramp. The implementation of the smoothing motion uses a smoothing function as defined in Eq. (2) to minimize model vibration at motion transition, and to yield discernible regions of constant pitch rate and motion acceleration. 


$$
\alpha(t)=\frac{\alpha_{m}}{2 B} \sum_{i=1}^{4}(-1)^{i+1} \ln \left\{\cosh \left[B\left(t-t_{i}\right) / t_{p}\right]\right\}, i=1,2,3,4
$$

where

$$
B=2 t_{p} / t_{a}
$$

The parameter $B$ is the ratio of pitch time $\left(t_{p}=\alpha_{m} / \alpha_{m}^{\prime}\right)$ to pitch acceleration time $\left(t_{a}=\alpha_{m}^{\prime} / \alpha_{m}^{\prime \prime}\right)$. The parameter $B$ can also be obtained by specifying parameters $\beta$ and $\gamma$. The $\beta$ is a ratio of smoothing angle to the maximum angle of attack; the $\gamma$ is the ratio of margin acceleration to the maximum acceleration during the smoothing region.

There are five phases in the wing motion, which includes start phase, pitch-up phase, hold phase, pitch-return phase, and relaxation phase. The duration for either pitch-up phase or pitch-return phase is the same. For force measurement, the duration of the start phase is one convective time; the hold duration is at least 130 convective times $(h>130)$ to ensure the flow reaching steady conditions at maximum pitch angle of $45^{\circ}$; the relaxation duration is about 30 convective times $(e>30)$ to return the flow to the undisturbed initial condition. For flow visualization and SPIV measurements, the time for relaxation is at least 30 convective time or pitch time.

The Velmex Rotary Table model B4832TS equipped with a stepper motor RK266-03 was used to implement the wing motion. There are 13 points within every smoothing angle. For all measurements the motion is repeated 60 times and phase averages are used to obtain mean values with relatively small uncertainty. For detail implementation of wing kinematics, see $\mathrm{Yu}$ et al. (2013) $)^{1}$, and $\mathrm{Yu}$ and Bernal $(2013)^{2}$.

\section{Direct Force Measurement and Data Processing}

For the force measurements the wing was attached to a sensor adapter through a hole-pattern aligned with the pitch axis, which coincident with the axis of a force sensor. The force sensor measures forces in three directions, which are chord-wise (x-axis), normal to the wing surface (y-axis) and span-wise direction (z-axis), and also the corresponding torques. Three pivot-axis locations were considered, including leading edge, mid-chord, and trailing edge. For both leading-edge and trailing-edge pivot axes, the wing has the same sensor configuration, but with 180degree The edge normal to the wing root chord (no sweep) is the pivot axis for all these cases and the wing is rotated $180^{\circ}$ from the LE to the TE pivot axis. The force transducer coordinate system is the same for the LE and ME pivot axis, and is rotated $180^{\circ}$ for the TE pivot axis. The leading-edge pivot and mid-chord pivot have the same coordinates of force transducer, but use wings with difference pitch-axis alignment.

The force sensor is a Nano 43 Force/Torque sensor manufactured by ATI Industrial Automation. The sensor's maximum calibrated load is $18 \mathrm{~N}$ and the resolution is $1 / 256 \mathrm{~N}$, in all three axes. The force sensor is attached to a rotary table Velmex (B4818TS) driven by a stepper motor, which has a resolution of $20 \mathrm{deg} / \mathrm{s}$ and maximum pitch rate of $200 \mathrm{deg} / \mathrm{s}$. Both the force sensor and the rotary table are located above the water surface. The wings are attached to the tool side of the sensor with an aluminum adapter designed to minimize the mass of the system. The mass attached to the sensor for all the wings are tabulated in Table 1, the total mass contributed to sensor is not more than 46.2 grams, which includes the wing itself, a sensor adapter and screws. Because of the small mass, inertia forces and static weight of the model are very small which makes static calibration unnecessary. The forces measured by the sensor $\left(F x\right.$ and $F y$ ) are first converted to axial and normal forces, $F_{A}$ and $F_{N}$, and then to components in the laboratory frame of reference using Eq. (2) - (5) for lift force $(L)$ and drag force $(D)$ and their corresponding force coefficients.

$$
\begin{gathered}
L=-F_{A} \sin \alpha+F_{N} \cos \alpha \\
D=F_{A} \cos \alpha+F_{N} \sin \alpha \\
C_{L}=L /\left(\rho U_{\infty}^{2} S / 2\right) \\
C_{D}=D /\left(\rho U_{\infty}^{2} S / 2\right)
\end{gathered}
$$

Data processing of force sensor signals includes application of a low-pass filter and a tare procedure. The lowpass filter is used to remove high frequency noise associated with structural vibrations and electronic/sensor noise, which is a zero-phase first-order two-path Butterworth filter. The filter cutoff frequency was determined using Fourier transform of the motion acceleration to retain $90 \%$ of spectrum content. A zero-phase 1st-order 2-path Butterworth filter was used to avoid phase distortion, to reduce spurious oscillations introduced by the filter, and to provide sufficient noise attenuation. For the pitch rate of $155 \mathrm{deg} / \mathrm{s}$, the cutoff frequency was $8.8 \mathrm{~Hz}$ to retain $90 \%$ of the frequency content of the motion acceleration, and the corresponding attenuation at the frequency of maximum energy content is $-5.39 \mathrm{~dB}$. It should be noted that the noise introduced by the rotary stage stepper motor is in the 
range of $100 \mathrm{~Hz}$ to $600 \mathrm{~Hz}$, and the wing vibration fundamental frequencies in air and in still water were determined by comparing with the configuration without wing and is also significantly higher than the filter cutoff frequency.

Tare procedures are generally necessary to remove inertia and weight contributions to the measured force due to the wing acceleration and position. These include static tares and dynamic tares. The static tares are measurements in air and still water at fixed angle from $0^{\circ}$ to maximum angle of attack $45^{\circ}$ with an increment of $3^{\circ}$. The dynamic tares are measurements in air with the same kinematics as in the flow experiments. In addition, the force measurements at fixed angle of attack in steady flow were conducted. The kinematics is similar to static tare measurements but with longer hold duration at the free-stream velocity of interest. In all force measurements the same filter cutoff frequency was used.

All measurements were repeated 60 times from $0^{\circ}$ to fixed angle of attack with sampling rate of $5000 \mathrm{~Hz}$, and ensemble averages are reported. It was found that the present wing configuration yields negligible static tares because of the very small mass of the wing and mounting hardware.

\section{Flow Visualization}

The flow visualization was conducted using a dye rake ( 7 probes with 1" spacing), a camera, two syringe pumps, and two different color food dyes (i.e. blue and red). Fig. 1 is an illustration of wing configuration used for dye visualization, a background white board with thickness of 0.24 " was employed to exclude distracting background features and reflection from the water surface. The injection rake was placed at $50 \%$ of wing span for all wing planforms, as shown by the red line in Fig. 7, and three mean chords upstream of the leading edge. Since the density of the dyes (ESCO Foods) is $1012 \mathrm{~kg} / \mathrm{m}^{3}$, they were mixed with alcohol to match the

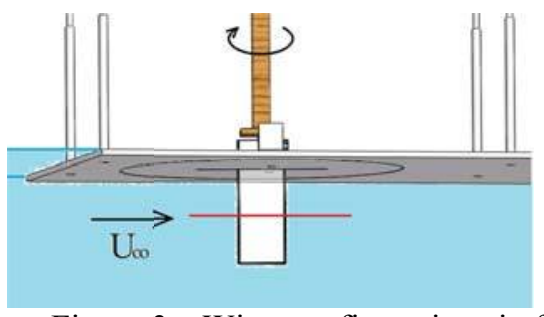

Figure 3 Wing configurations in flow visualization water density, $998 \mathrm{~kg} / \mathrm{m}^{3}$. The images were recorded using Nikon D3100 camera at a frame rate of $30 \mathrm{~Hz}$. Images in the side and top views are reported. The side view provides the field of view of flow about the wing chord; the top view gives the field of view of flow about the wing span.

\section{E. PIV Measurements}

Conventional 2D PIV and lens-shifted Stereo PIV were used to measure the unsteady flow about the wings. The lens-shifted Stereo PIV system is illustrated in Figure 4 for coordinates on X-Z plane and Y-Z plane, respectively. For a detailed description of the PIV system, PIV data acquisition and PIV data processing the reader is referred to $\mathrm{Yu}{ }^{3}$. In a typical test the magnification used was $13 \mathrm{px} / \mathrm{mm}$ and the particle displacement between images approximately $8 \mathrm{px}$.

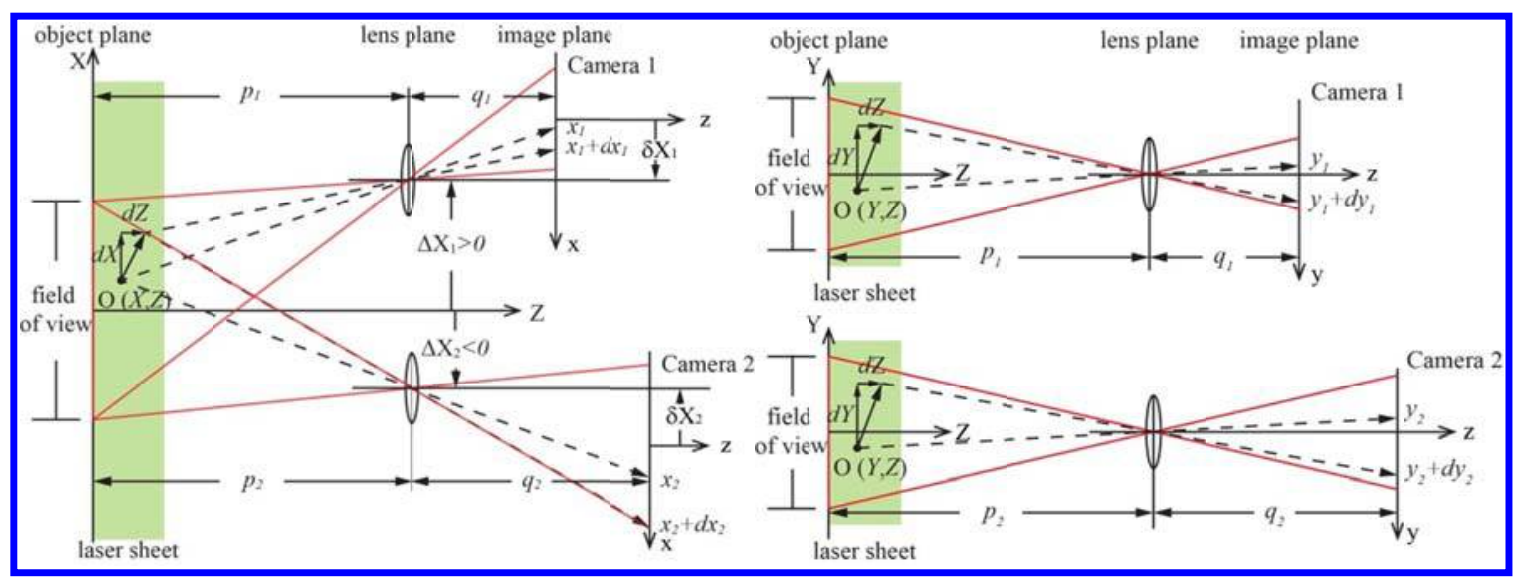

Figure 4 Schematics of a lens shifted Stereo PIV geometry in the x-z plane. 
The PIV images were processed using in-house developed MATLAB software which uses a cross-correlation algorithm to determine the particle displacement with subpixel resolution. A two-pass algorithm is used to improve the resolution of the measurement. Results describe here are phase averages of approximately 60 images.

\section{Results}

\section{A. Flow Visualization}

Figure 5 shows flow visualization results at $\mathrm{K}=0.39$ and 0.06 . Also shown at the bottom of the figure are the corresponding lift coefficient time history.
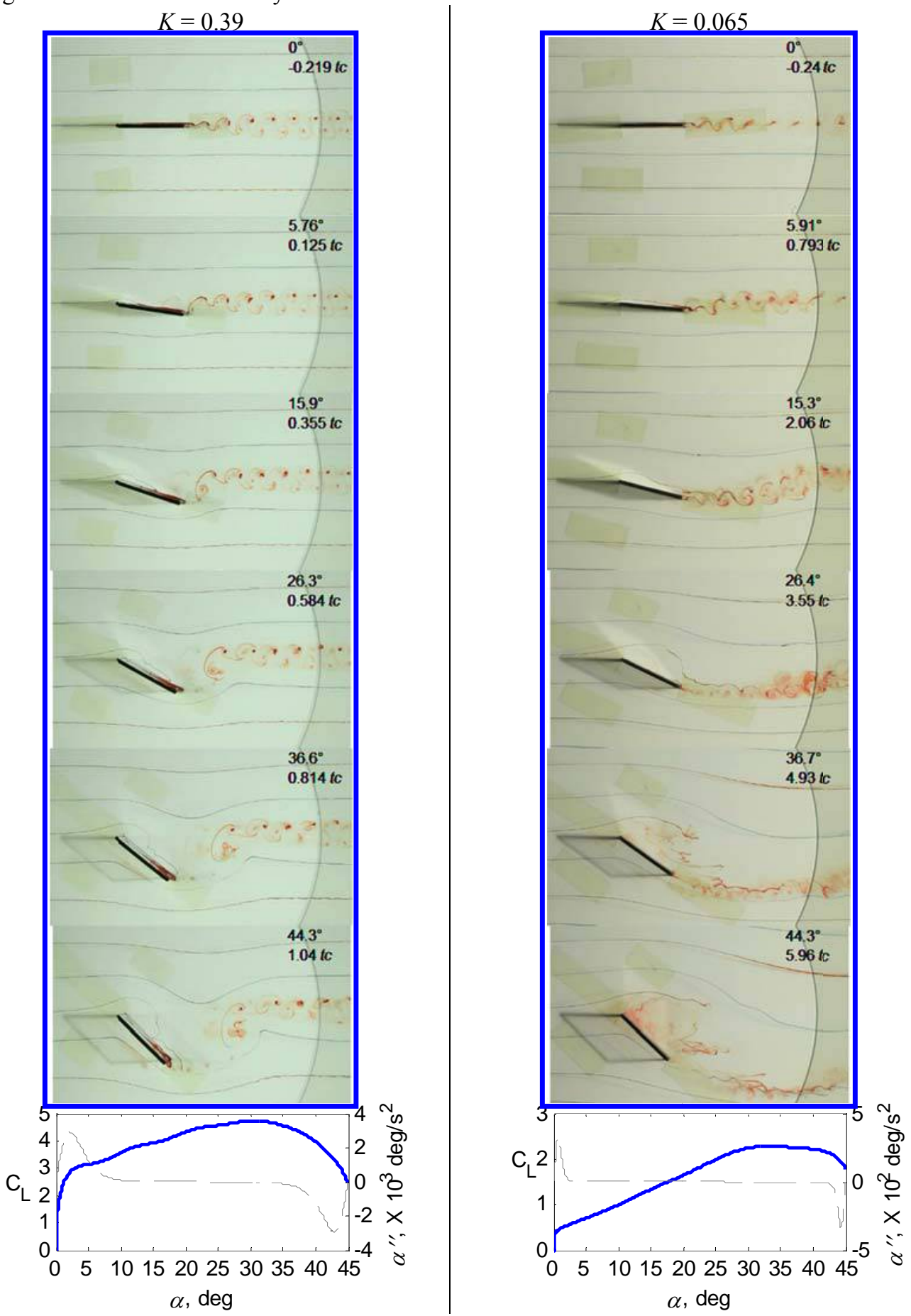

Figure 5. Flow visualization during pitch-up phase at $\mathrm{K}=0.39$ and 0.06 . 
Several features are apparent in the evolution of the flow:

1 At an early stage and higher reduced pitch rate, the flow is essentially two dimensional.

2 At high pitch rate the leading edge vortex does not appear to form until late in the wing motion.

3 The starting vortex is larger at higher reduced pitch rate and forms at lower angle of attack. The formation of the starting vortex may be associated with the rapid wing motion.

4 At lower reduced pitch rate the leading-edge vortex forms at lower angle of attack. However, this leadingedge vortex detaches at high angle of attack and three-dimensional flow develops in the wake, leading to the saturation of forces.
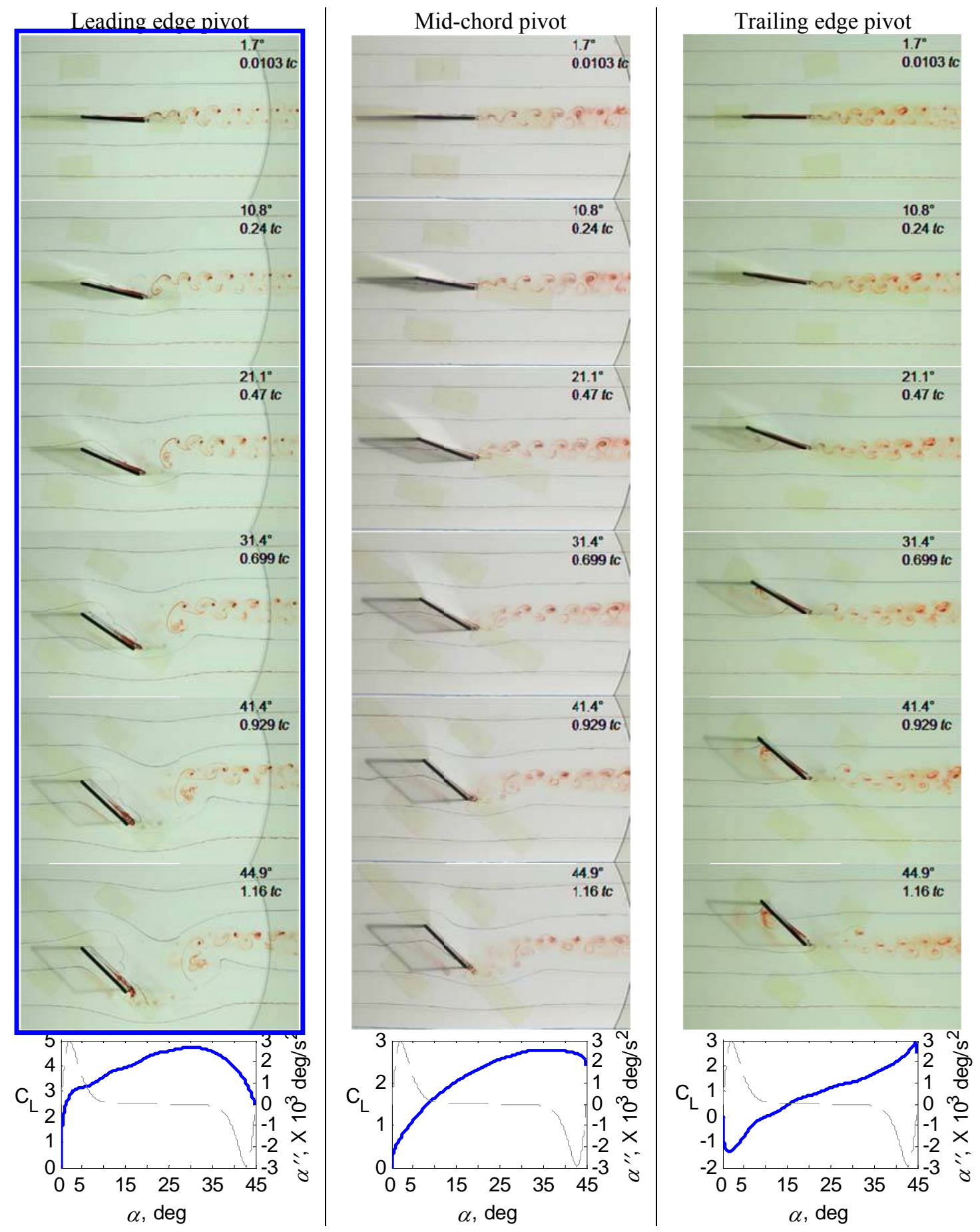

Figure 6. Effect of pivot axis location in side view during pitch-up phase.

American Institute of Aeronautics and Astronautics 
Figure 6 Figure 6show the effect of pivot axis location on the flow field for a rectangular wing at $\mathrm{K}=0.39$. Also shown at the bottom are the corresponding lift force time history during the motion. The center streakline shows attached flow over the wing and Kármán vortex shedding in the wake at low angle of attack. As the angle of attack is increased to $21^{\circ}$ a starting vortex forms in the near wake. For trailing-edge-pivot wing, a leading edge vortex forms at about quarter-chord on the windward surface. In the near wake, there is a vortex with clockwise rotation shed from the leeward surface at trailing edge, different from the typical starting vortex with counterclockwise rotation. The deflections of outer streaklines by leading-edge-pivot and trailing-edge-pivot wings are in opposite direction. The deflection of the outer streaklines by mid-chord-pivot wing indicates the presence of a weak starting vortex in the wake; it is unclear that there is a vortex formed at the leading edge. Detailed review of the video shows that the typical starting vortex at the trailing edge forms at $6^{\circ}\left(0.125 t_{c}\right)$ and $16^{\circ}\left(0.355 t_{c}\right)$ by leading-edge-pivot wing and mid-chord-pivot wing, respectively; the "reverse" starting vortex was formed at $16^{\circ}\left(0.355 t_{c}\right)$ by trailingedge-pivot wing. As the angle of attack is increased to $45^{\circ}$, the starting vortex by leading-edge-pivot wing convects further downstream; however, the starting vortex by trailing-edge-pivot wing grows in size at the same location, as shown by the increase of red-dye concentration.

\section{B. Direct Force Measurements}

Here we highlight some significant result on the effect of pitch rate and pivot location on the unsteady force acting on rectangular aspect ratio 4 wings.
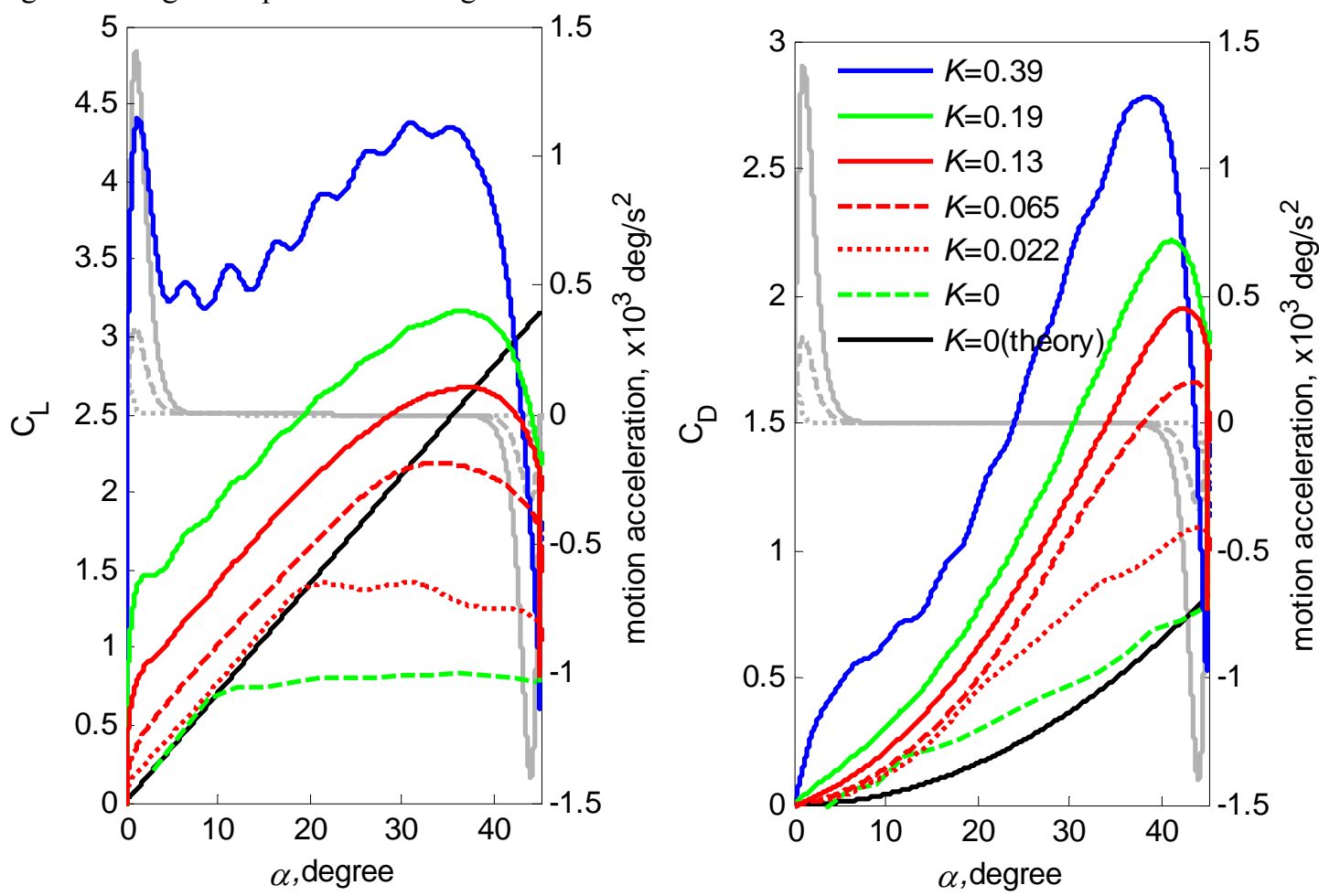

Figure 7. Force coefficients as a function of angle of attack using rectangular wing at leading edge pivot (left) lift coefficient (right) drag coefficient

Figure 7 shows the lift and drag coefficients of the rectangular wing pitching at the leading edge. At the lowest reduced pitch rate (i.e. $K=0.022$ ) the lift coefficient follows the linear potential flow theory result closely up to an angle of attack of 20 degrees, which corresponds to the steady stall angle of attack. The slope of lift-curve is slightly depressed due to the onset of rotation; this feature was noticed by Jumper et al. ${ }^{4}$ for NACA0015 at $K<0.01$. The drag coefficients is significantly higher than the prediction by the lifting-line theory, a better estimate consistent with the relatively small value of the leading edge. suction is assumed that the aerodynamic force is normal to the plate, which follows the dashed line in the right panel of Figure 7. For larger angles of attack the drag coefficient increases while the lift coefficient remains approximately constant which implies smaller L/D values. These data suggest that for $K<0.022$ the flow is quasi-steady and the lifting-line theory provides good estimates of the lift coefficients up to the stall angle of attack. In this regime the drag coefficient is under predicted by the lifting line theory by a factor of 
two. Thus the lifting-line theory appears to correctly account for finite wing effects in the slope of the lift curve (i.e. a $33 \%$ reduction of the lift coefficient according to the infinite wing), but fails to account for the significant reduction in leading edge suction which results in largest drag coefficients compared to induced drag calculations.

For $K>0.022$ unsteady effects are important; the flow visualization data revealed two-dimensional flow is more pronounced as the reduced pitch rate increases. There are two main effects for the lift coefficient: a non-circulatory peak during wing acceleration, and pitch rate effects within regions of constant pitch rate, the lift coefficients shift upward with same amount as reduced pitch rate increases. Similar effects were documented for 2D flow by Granlund et al. ${ }^{5}$ Both effects are very strong at much higher reduced pitch rate $K=0.39$. As noted earlier, noncirculatory effects produce a positive spike at the start of pitch-up phase and a negative spike at the end of pitch-up phase within acceleration region. This force spikes are associated with the formation of the starting vortex and will be discussed in next chapter. The shift in the lift coefficient curve due to pitch-rate effect is similar to mean camber effects as would be expected from linear potential flow theory. Unsteady effects result in an increase in drag coefficient. At $K=0.39$ there is a significant increase of drag at small angle of attack during the acceleration portion of the pitching motion.
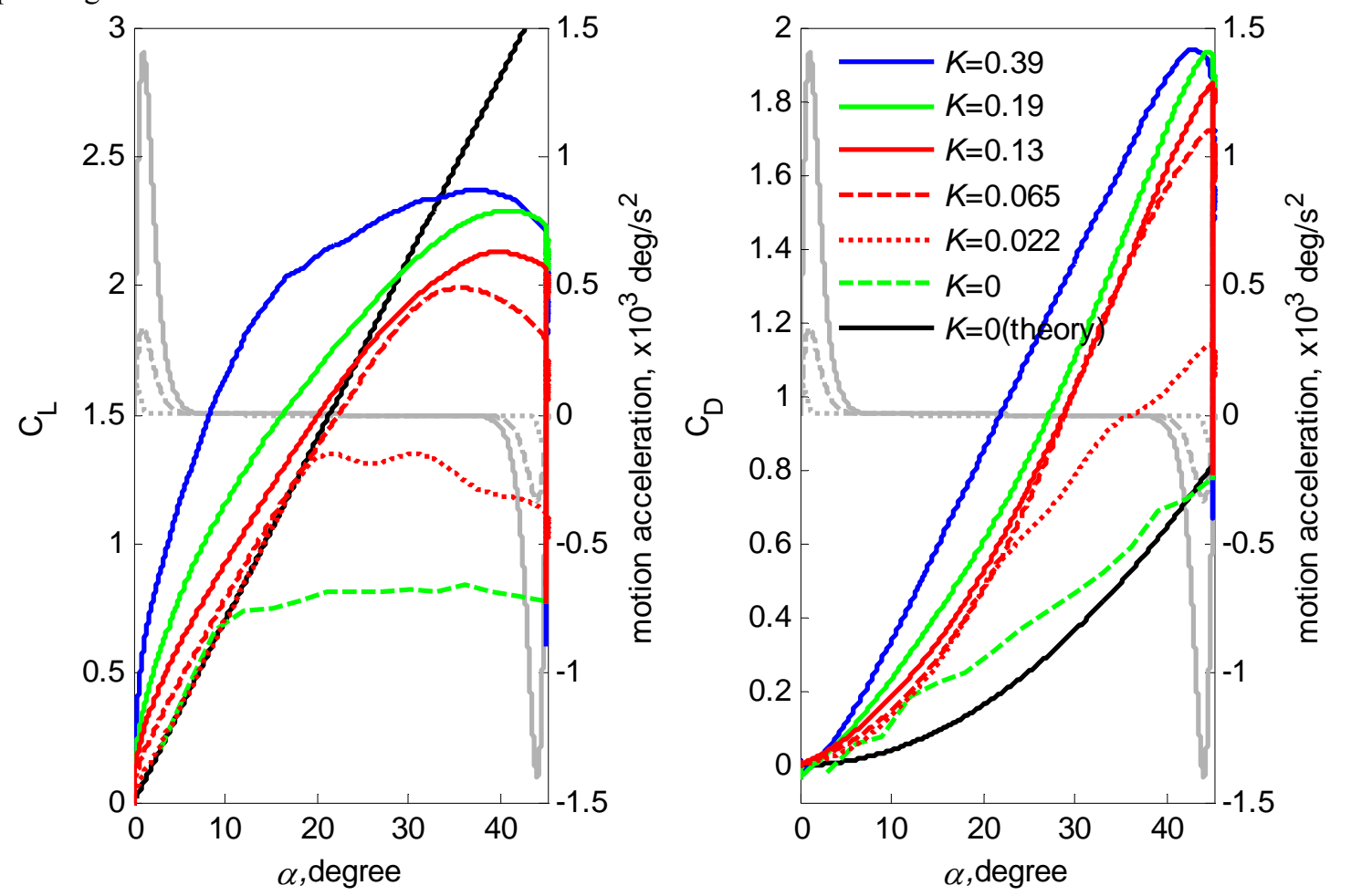

Figure 8 Force coefficients as a function of angle of attack using rectangular wing at mid-chord pivot (left) lift coefficient (right) drag coefficient

Figure 8 shows the lift and drag coefficients at mid-chord pivot axis as a function of angle of attack. At the lowest reduced pitch rate $(K=0.022)$ the lift coefficient follows the theoretical results closely up to an angle of attack of 20 degrees. The drag coefficient follows similar trends as for the leading edge pivot axis. These data confirm the conclusion that for $K<0.022$ the flow is quasi-steady and the lifting line theory provides good estimates of the lift coefficient up to the stall angle of attack.

For values of $K>0.022$ unsteady effects are important. However the trends are different compared to leading edge pivot. In this case non-circulatory effects are very small. Pitch rate effects between the initial and final acceleration are much stronger at $K>0.065$, which increases the lift coefficient at small angles of attack. Within the constant pitch rate region, the lift coefficient curves are likely shifting upward as reduced pitch rate increases, but not with the same amount for leading edge pivot axis. The drag coefficient shows small change in the drag coefficient for $K<0.39$. The case $K=0.39$ shows larger effects in both the lift and drag coefficients.

Figure 9 shows the lift and drag coefficients for trailing edge pivot axis. At the lowest reduced pitch rate $(K=$ 0.022 ) the lift coefficient and drag coefficient show similar quasi steady behavior as for other pivot axes. At higher reduced pitch rate the lift coefficients are less than the estimation from the lifting line theory, and not very different 
compared to the results at $K=0.022$, except for $K=0.39$. Non-circulatory effects result in a negative lift coefficient at the beginning of pitch and a positive spike at the end of rotation. The magnitude of the spike is moderate except for the $K=0.39$ case. For $K=0.39$ the initial acceleration persist well beyond the end of the acceleration period where the lift coefficient is negative. The drag coefficient is also negative in this region.
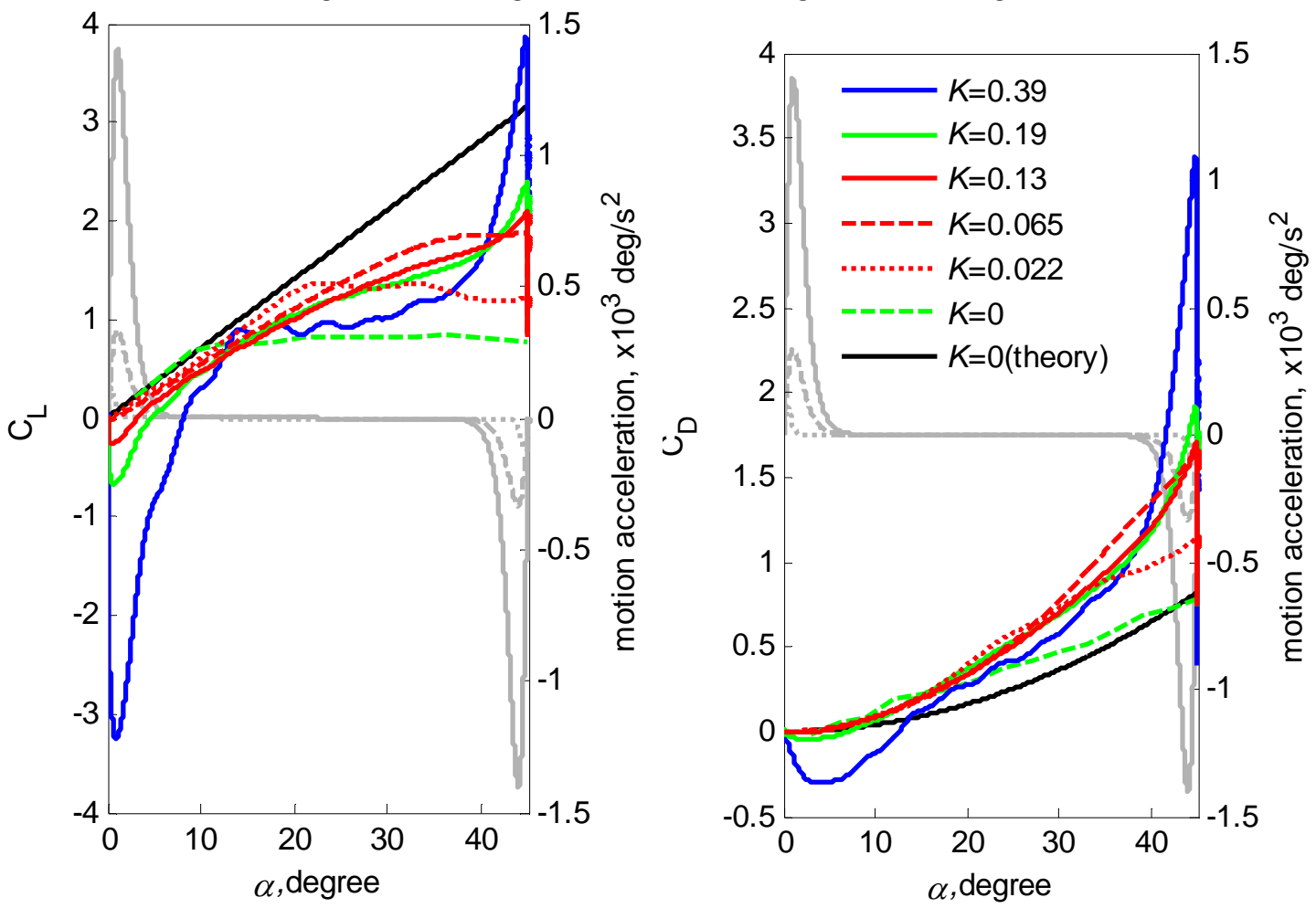

Figure 9. Force coefficients as a function of angle of attack using rectangular wing at trailing edge pivot (left) lift coefficient (right) drag coefficient

\section{PIV Velocity Measurement}

Error! Reference source not found. 10 and 11 show vorticity flow fields of a rectangular wing pitching at the leading edge for reduced pitch rates $K=0.39$ and 0.065 , respectively. The vorticity fields at three span locations are considered in attempt to character the perspective of vortical flow during the pitch-up phase, which are $50 \%$ span, $75 \%$ span, and $100 \%$ span.

For $K=0.39$ at $50 \%$ span, there is a starting vortex formed at the trailing edge; the deflection of the streamlines toward the starting vortex indicates its presence at an angle of attack $7.5^{\circ}$ in Figure. Similar deflected streaklines were introduced to identify the occurrence of starting vortex for flow visualization data (Figure 5). The force data showed that the normal force increases rapidly up to this phase angle. As the angle of attack is increased to $22.5^{\circ}$, the starting vortex convects downstream; the negative vorticity at the leading edge becomes much stronger with positive out-of-plane velocity around the vortex core. The force data showed an increase of normal force with a slope similar to those with lower reduced pitch rates in the same free-stream flow, this angle of attack is well beyond the steady stall angle of attack (i.e. about $12^{\circ}$ ). As the angle of attack is increased to $37.5^{\circ}$, the negative vorticity at leading edge forms a well-known leading-edge vortex, which is identified according to the presence of streamline circulation core. This leading-edge vortex circulation on the leeward surface and forms positive vorticity in a cavity underneath the neck of the leading-edge vortex; this positive vorticity forms a secondary vortex. As the angle of attack is increased to about $45^{\circ}$, the starting vortex is about one chord downstream from the trailing edge; the leading-edge vortex grows in size at the forward portion of the chord on the leeward surface. At this phase, the force data decreased in accordance with the wing deceleration. The span variation of the flow evolution is small, except the flow evolution at the wing tip. At higher angle of attack, the starting vortex formed at an earlier stage does not move downstream but interacts with out-of-plane velocity field at the trailing edge. The starting vortex becomes a portion of positive vorticity above the negative vorticity on the leeward surface. The out-of-plane velocity field 
shows an increase of wingtip circulation with increasing angle of attack; this wingtip circulation stays close to the wing, introducing significant drag on the wing.

For $K=0.065$ at $50 \%$ span, the starting vortex is indiscernible at the lower angle of attack $7.5^{\circ}$; there are few trailing-edge vortices in the near wake. From the force data, there is not normal force spike within this angle of attack, which indicates the importance of the starting vortex to the normal force spike. The negative vorticity thickness becomes much thicker as the angle of attack is increased to $15^{\circ}$ with more trailing edge vortices in the wake. This is the common feature before leading-edge vortex is present. The leading-edge vortex is discernible at an angle of attack $22.5^{\circ}$, which is much earlier than the other cases with higher reduced pitch rate. This leading-edge vortex covers entire wing chord and grows in size as increasing angle of attack; the secondary vortex is introduced during the growth of the leading-edge vortex as well. Moreover, the spanwise variation is more pronounced than the other higher reduced pitch rates in both vorticity and out-of-plane velocity field. At the wingtip, the onset of wingtip circulation begines from the negative out-of-plane velocity about trailing edge and positive out-of-plane velocity on the leeward surface, similar to the other cases with higher reduced pitch rates; however, the wingtip circulation convects downstream much quicker for a given angle of attack due to larger time ratio $t_{p} / t_{c}$

\section{Conclusion}

Flow visualization, unsteady aerodynamic force, and PIV flow measurements have been reported for pitching flat-plate wings with constant pitch rates to a maximum angle of attack of 45 degrees. The wings had an aspect ratio equal to 4 and rectangular, trapezoidal, and triangular planforms. Changing either wing pitch rate or free stream flow results in a change of reduced pitch rate within an interval of $0.022 \leq K \leq 0.39$ in a parameter space of Stokes number $(S t)$ and Reynolds number $(R e)$. Also the case of $R e=0$ (i.e. $K=\infty$ ) and $K=0$ in $R e=8.9 \mathrm{k}$ have been documented to gain additional insight on the nature of non-circulatory effect and unsteady effect. The conclusions of this study are summarized in the following:

1 At high pitch rates the flow is substantially two dimensional except for a small region near the wing tip.

2 Three dimensional flow developments have two basic forms. Large scale swirl associated with tip vortices and other streamwise vorticity, and small scale three dimensional flow feature of size comparable to the thin shear layers that form at the separation point possibly due to fast instabilities of the shear layers.

3 Three dimensional flow development occurs at very fast time scales. Lift curve slope during the constant pitch rate region is significantly reduced compared to the two-dimensional flow value associated which is attributed to very rapid three-dimensional flow development.

4 Reduced rate effects are dominant in force generation during the pitch up motion, which in turn are very sensitive to pivot axis location.

a. For leading edge pivot axis very large lift coefficient are measured at high reduced pitch rates, before the development of large Leading Edge Vortex. A stonger trailing edge vortex appears to form at this condition

b. For mid-cord pivot lift generation is significantly less than for leading edges pivot.

c. For trailing edge pivot rotation rate effect contributes negative lift.

5 Non-circulatory inertia effects are confined to the transient pitch acceleration at the start and end of the wing motion. Smalls are observed at no flow conditions.

\section{Acknowledgments}

The authors would like to thank Dr. Michael Ol and Dr. Kenneth Granlund from the Air Force Research Laboratory (AFRL) for many fruitful discussions of the research reported in this paper. The work was sponsored in part by the Air Force Office of Scientific Research's Multidisciplinary University Research Initiative (MURI), contract number FA9550-07-1-0547 and by the Michigan/AFRL Collaborative Center in Aeronautical Sciences. 

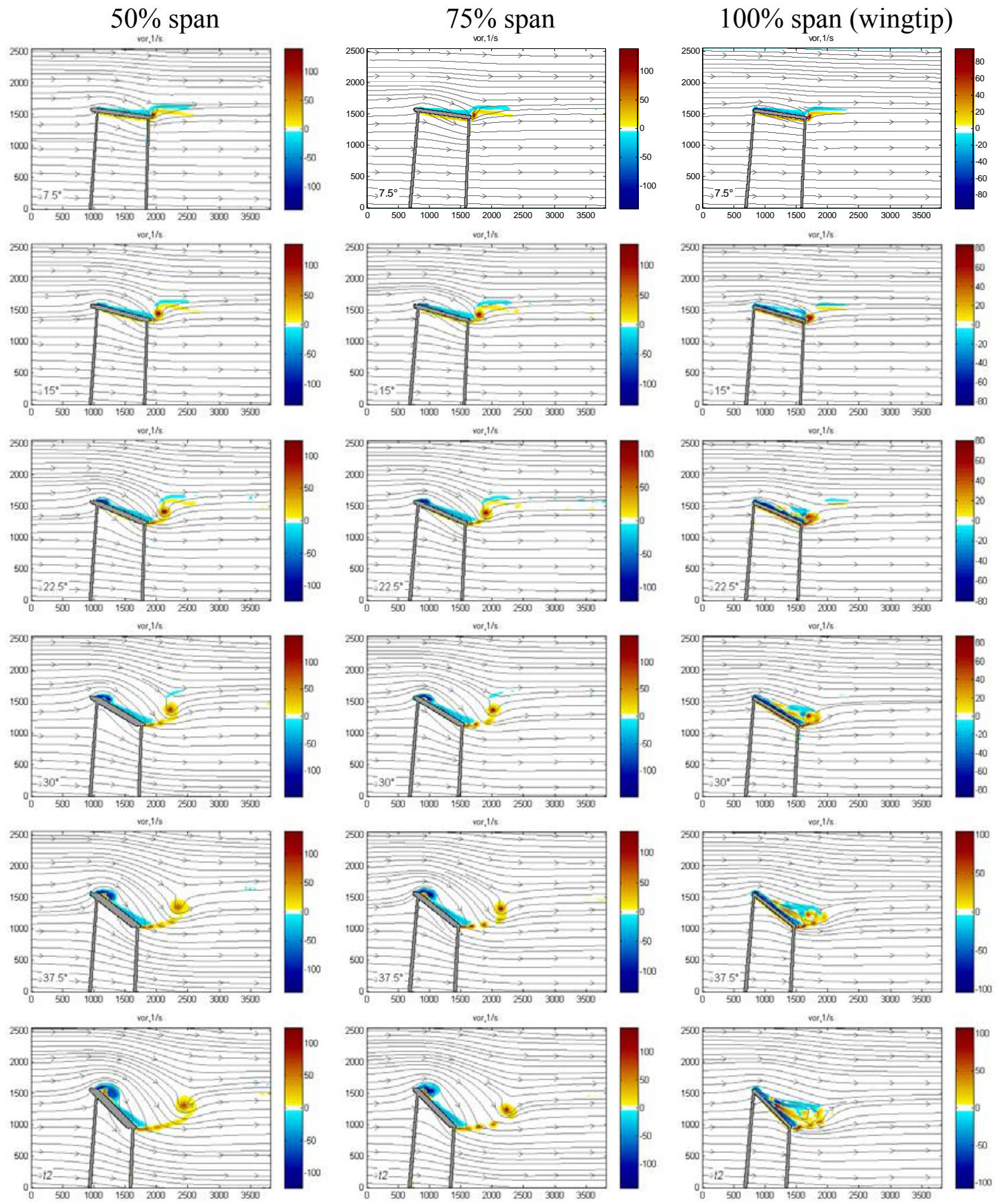

Figure 10. PIV instantaneous vorticity field and streamlines for $K=0.39$ at leading edge pivot. 


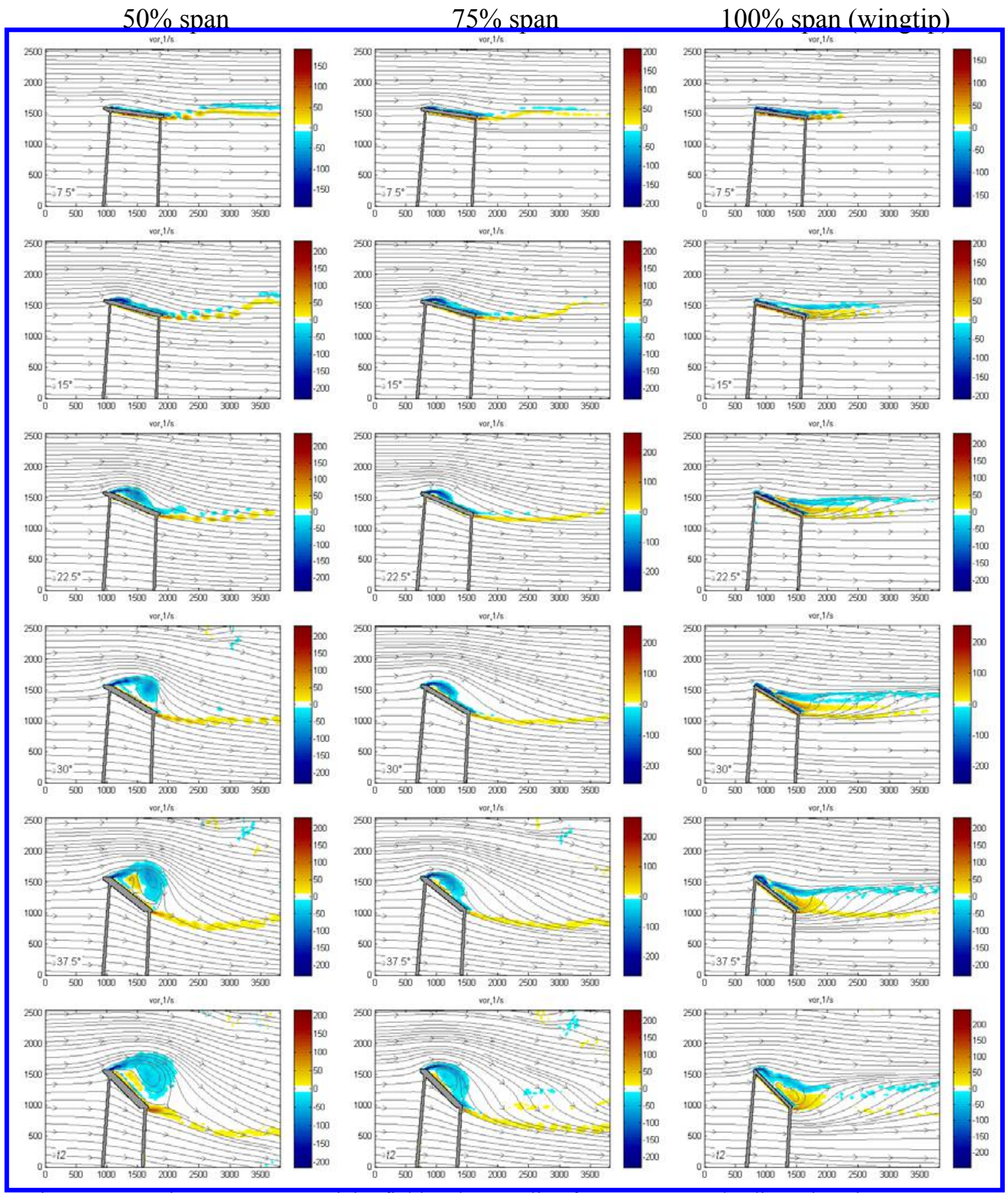

Figure 11 PIV instantaneous vorticity field and streamline for $K=0.065$ at leading edge pivot.

\section{References}

${ }^{1}$ Yu, H.T., Bernal, L.P, and Morrison, C., "Experimental Investigation of Pitch Ramp-Hold-Return Motion of Flat Plates at Low Reynolds Number", AIAA Paper 2012 - 51, Jan. 2012.

${ }^{2} \mathrm{Yu}$, H.T. and Bernal, L.P, "Effect of Pivot Point on Aerodynamic Force and Vortical Structure of Pitching Flat Plate Wings", AIAA Paper 2013 - 0792, Jan. 2013.

${ }^{3} \mathrm{Yu}, \mathrm{H}-\mathrm{T}$. "Unsteady Aerodynamics of Pitching Flat Plate Wings," PhD Thesis, University of Michigan, May 2014.

${ }^{4}$ Jumper, E.J., Schreck, S.J., and Dimmick, R.L., "Lift-Curve Characteristics for an Airfoil Pitching at Constant Rate", Journal of Aircraft, Vol. 2, No. 10, 1987, pp. 680-687.

${ }^{5}$ Granlund, K. O., O1, M. V., and Bernal, L. P., “Unsteady Pitching Flat Plates,” Journal of Fluid Mechanics, Vol. 733, R5, 2013. 